\title{
Search for Supersymmetry via Associated Production of Charginos and Neutralinos in Final States with Three Leptons
}

V. M. Abazov, ${ }^{35}$ B. Abbott, ${ }^{72}$ M. Abolins, ${ }^{63}$ B. S. Acharya, ${ }^{29}$ M. Adams, ${ }^{50}$ T. Adams, ${ }^{48}$ M. Agelou, ${ }^{18}$ J.-L. Agram, ${ }^{19}$ S. H. Ahn,${ }^{31}$ M. Ahsan,${ }^{57}$ G. D. Alexeev,${ }^{35}$ G. Alkhazov, ${ }^{39}$ A. Alton,${ }^{62}$ G. Alverson, ${ }^{61}$ G. A. Alves, ${ }^{2}$ M. Anastasoaie, ${ }^{34}$ T. Andeen, ${ }^{52}$ S. Anderson, ${ }^{44}$ B. Andrieu, ${ }^{17}$ Y. Arnoud, ${ }^{14}$ A. Askew, ${ }^{48}$ B. Åsman, ${ }^{40}$ A. C. S. Assis Jesus, ${ }^{3}$ O. Atramentov,${ }^{55}$ C. Autermann, ${ }^{21}$ C. Avila, ${ }^{8}$ F. Badaud,${ }^{13}$ A. Baden, ${ }^{59}$ B. Baldin,${ }^{49}$ P. W. Balm,${ }^{33}$ S. Banerjee,${ }^{29}$ E. Barberis,${ }^{61}$ P. Bargassa, ${ }^{76}$ P. Baringer ${ }^{56}$ C. Barnes, ${ }^{42}$ J. Barreto, ${ }^{2}$ J. F. Bartlett, ${ }^{49}$ U. Bassler, ${ }^{17}$ D. Bauer, ${ }^{53}$ A. Bean,${ }^{56}$ S. Beauceron, ${ }^{17}$ M. Begel,${ }^{68}$ A. Bellavance, ${ }^{65}$ S. B. Beri, ${ }^{27}$ G. Bernardi, ${ }^{17}$ R. Bernhard, ${ }^{49}, *$ I. Bertram, ${ }^{41}$ M. Besançon, ${ }^{18}$ R. Beuselinck,${ }^{42}$ V. A. Bezzubov, ${ }^{38}$ P.C. Bhat, ${ }^{49}$ V. Bhatnagar, ${ }^{27}$ M. Binder, ${ }^{25}$ C. Biscarat, ${ }^{41}$ K. M. Black, ${ }^{60}$ I. Blackler, ${ }^{42}$ G. Blazey, ${ }^{51}$ F. Blekman, ${ }^{33}$ S. Blessing,${ }^{48}$ D. Bloch,${ }^{19}$ U. Blumenschein, ${ }^{23}$ A. Boehnlein,${ }^{49}$ O. Boeriu, ${ }^{54}$ T. A. Bolton, ${ }^{57}$ F. Borcherding, ${ }^{49}$ G. Borissov, ${ }^{41}$ K. Bos,${ }^{33}$ T. Bose,${ }^{67}$ A. Brandt,${ }^{74}$ R. Brock,${ }^{63}$ G. Brooijmans, ${ }^{67}$ A. Bross, ${ }^{49}$ N. J. Buchanan, ${ }^{48}$ D. Buchholz, ${ }^{52}$ M. Buehler, ${ }^{50}$ V. Buescher, ${ }^{23}$ S. Burdin, ${ }^{49}$ T. H. Burnett, ${ }^{78}$ E. Busato, ${ }^{17}$ C. P. Buszello, ${ }^{42}$ J. M. Butler, ${ }^{60}$ J. Cammin, ${ }^{68}$ S. Caron, ${ }^{33}$ W. Carvalho, ${ }^{3}$ B. C. K. Casey,${ }^{73}$ N. M. Cason, ${ }^{54}$ H. Castilla-Valdez, ${ }^{32}$ S. Chakrabarti, ${ }^{29}$ D. Chakraborty,${ }^{51}$ K. M. Chan, ${ }^{68}$ A. Chandra, ${ }^{29}$ D. Chapin,${ }^{73}$ F. Charles, ${ }^{19}$ E. Cheu, ${ }^{44}$ D. K. Cho, ${ }^{60}$ S. Choi, ${ }^{47}$ B. Choudhary, ${ }^{28}$ T. Christiansen, ${ }^{25}$ L. Christofek, ${ }^{56}$ D. Claes, ${ }^{65}$ B. Clément, ${ }^{19}$ C. Clément, ${ }^{40}$ Y. Coadou, ${ }^{5}$ M. Cooke, ${ }^{76}$ W. E. Cooper, ${ }^{49}$ D. Coppage, ${ }^{56}$ M. Corcoran, ${ }^{76}$ A. Cothenet, ${ }^{15}$ M.-C. Cousinou, ${ }^{15}$ B. Cox,${ }^{43}$

S. Crépé-Renaudin, ${ }^{14}$ D. Cutts, ${ }^{73}$ H. da Motta, ${ }^{2}$ B. Davies,${ }^{41}$ G. Davies, ${ }^{42}$ G. A. Davis,${ }^{52}$ K. De,${ }^{74}$ P. de Jong, ${ }^{33}$ S. J. de Jong, ${ }^{34}$ E. De La Cruz-Burelo, ${ }^{32}$ C. De Oliveira Martins, ${ }^{3}$ S. Dean, ${ }^{43}$ J. D. Degenhardt, ${ }^{62}$ F. Déliot, ${ }^{18}$ M. Demarteau ${ }^{49}$ R. Demina, ${ }^{68}$ P. Demine, ${ }^{18}$ D. Denisov, ${ }^{49}$ S. P. Denisov, ${ }^{38}$ S. Desai, ${ }^{69}$ H. T. Diehl,${ }^{49}$ M. Diesburg, ${ }^{49}$ M. Doidge, ${ }^{41}$ H. Dong, ${ }^{69}$ S. Doulas, ${ }^{61}$ L. V. Dudko, ${ }^{37}$ L. Duflot, ${ }^{16}$ S. R. Dugad,${ }^{29}$ A. Duperrin, ${ }^{15}$ J. Dyer,${ }^{63}$ A. Dyshkant,${ }^{51}$

M. Eads,${ }^{51}$ D. Edmunds, ${ }^{63}$ T. Edwards, ${ }^{43}$ J. Ellison, ${ }^{47}$ J. Elmsheuser, ${ }^{25}$ V. D. Elvira, ${ }^{49}$ S. Eno, ${ }^{59}$ P. Ermolov, ${ }^{37}$ O. V. Eroshin, ${ }^{38}$ J. Estrada,${ }^{49}$ H. Evans,${ }^{67}$ A. Evdokimov, ${ }^{36}$ V. N. Evdokimov, ${ }^{38}$ J. Fast, ${ }^{49}$ S. N. Fatakia, ${ }^{60}$ L. Feligioni, ${ }^{60}$ A. V. Ferapontov, ${ }^{38}$ T. Ferbel, ${ }^{68}$ F. Fiedler, ${ }^{25}$ F. Filthaut, ${ }^{34}$ W. Fisher, ${ }^{66}$ H. E. Fisk, ${ }^{49}$ I. Fleck, ${ }^{23}$ M. Fortner, ${ }^{51}$ H. Fox,${ }^{23}$ S. Fu, ${ }^{49}$ S. Fuess, ${ }^{49}$ T. Gadfort ${ }^{78}$ C. F. Galea, ${ }^{34}$ E. Gallas, ${ }^{49}$ E. Galyaev, ${ }^{54}$ C. Garcia, ${ }^{68}$ A. Garcia-Bellido, ${ }^{78}$ J. Gardner,${ }^{56}$ V. Gavrilov, ${ }^{36}$ P. Gay, ${ }^{13}$ D. Gelé, ${ }^{19}$ R. Gelhaus,${ }^{47}$ K. Genser, ${ }^{49}$ C. E. Gerber, ${ }^{50}$ Y. Gershtein, ${ }^{48}$ D. Gillberg,,${ }^{5}$ G. Ginther,${ }^{68}$ T. Golling, ${ }^{22}$ N. Gollub, ${ }^{40}$ B. Gómez, ${ }^{8}$ K. Gounder, ${ }^{49}$ A. Goussiou, ${ }^{54}$ P. D. Grannis,${ }^{69}$ S. Greder, ${ }^{3}$ H. Greenlee, ${ }^{49}$

Z. D. Greenwood, ${ }^{58}$ E. M. Gregores, ${ }^{4}$ Ph. Gris, ${ }^{13}$ J.-F. Grivaz, ${ }^{16}$ L. Groer, ${ }^{67}$ S. Grünendahl, ${ }^{49}$ M. W. Grünewald, ${ }^{30}$ S. N. Gurzhiev ${ }^{38}$ G. Gutierrez,${ }^{49}$ P. Gutierrez, ${ }^{72}$ A. Haas, ${ }^{67}$ N. J. Hadley ${ }^{59}$ S. Hagopian, ${ }^{48}$ I. Hall, ${ }^{72}$ R. E. Hall, ${ }^{46}$ C. Han,${ }^{62}$ L. Han, ${ }^{7}$ K. Hanagaki, ${ }^{49}$ K. Harder,${ }^{57}$ A. Harel, ${ }^{26}$ R. Harrington, ${ }^{61}$ J. M. Hauptman, ${ }^{55}$ R. Hauser, ${ }^{63}$ J. Hays, ${ }^{52}$ T. Hebbeker, ${ }^{21}$ D. Hedin, ${ }^{51}$ J. M. Heinmiller ${ }^{50}$ A. P. Heinson, ${ }^{47}$ U. Heintz,${ }^{60}$ C. Hensel, ${ }^{56}$ G. Hesketh, ${ }^{61}$ M. D. Hildreth, ${ }^{54}$ R. Hirosky, ${ }^{77}$ J. D. Hobbs,${ }^{69}$ B. Hoeneisen, ${ }^{12}$ M. Hohlfeld, ${ }^{24}$ S. J. Hong, ${ }^{31}$ R. Hooper ${ }^{73}$ P. Houben, ${ }^{33}$ Y. Hu, ${ }^{69}$ J. Huang,${ }^{53}$ V. Hynek, ${ }^{9}$ I. Iashvili, ${ }^{47}$ R. Illingworth, ${ }^{49}$ A. S. Ito, ${ }^{49}$ S. Jabeen, ${ }^{56}$ M. Jaffré, ${ }^{16}$ S. Jain, ${ }^{72}$ V. Jain, ${ }^{70}$ K. Jakobs, ${ }^{23}$ A. Jenkins,${ }^{42}$ R. Jesik, ${ }^{42}$ K. Johns, ${ }^{44}$ M. Johnson, ${ }^{49}$ A. Jonckheere ${ }^{49}$ P. Jonsson, ${ }^{42}$ A. Juste ${ }^{49}$ D. Käfer ${ }^{21}$ S. Kahn, ${ }^{70}$ E. Kajfasz,${ }^{15}$ A. M. Kalinin, ${ }^{35}$ J. Kalk, ${ }^{63}$ D. Karmanov,${ }^{37}$ J. Kasper, ${ }^{60}$ D. Kau ${ }^{48}$ R. Kaur,${ }^{27}$ R. Kehoe, ${ }^{75}$ S. Kermiche, ${ }^{15}$ S. Kesisoglou, ${ }^{73}$ A. Khanov ${ }^{68}$ A. Kharchilava, ${ }^{54}$ Y. M. Kharzheev,${ }^{35}$ H. Kim, ${ }^{74}$ T. J. Kim,${ }^{31}$ B. Klima, ${ }^{49}$ J. M. Kohli, ${ }^{27}$ M. Kopal, ${ }^{72}$ V. M. Korablev, ${ }^{38}$ J. Kotcher ${ }^{70}$ B. Kothari, ${ }^{67}$ A. Koubarovsky, ${ }^{37}$ A. V. Kozelov, ${ }^{38}$ J. Kozminski, ${ }^{63}$ A. Kryemadhi, ${ }^{77}$ S. Krzywdzinski, ${ }^{49}$ Y. Kulik, ${ }^{49}$ A. Kumar, ${ }^{28}$ S. Kunori, ${ }^{59}$ A. Kupco, ${ }^{11}$ T. Kurča ${ }^{20}$ J. Kvita, ${ }^{9}$ S. Lager, ${ }^{40}$ N. Lahrichi, ${ }^{18}$ G. Landsberg ${ }^{73}$ J. Lazoflores ${ }^{48}$ A.-C. Le Bihan, ${ }^{19}$ P. Lebrun, ${ }^{20}$ W. M. Lee, ${ }^{48}$ A. Leflat,${ }^{37}$ F. Lehner, ${ }^{49, *}$

C. Leonidopoulos, ${ }^{67}$ J. Leveque, ${ }^{44}$ P. Lewis,${ }^{42}$ J. Li ${ }^{74}$ Q. Z. Li, ${ }^{49}$ J. G. R. Lima,${ }^{51}$ D. Lincoln, ${ }^{49}$ S. L. Linn, ${ }^{48}$ J. Linnemann, ${ }^{63}$ V. V. Lipaev, ${ }^{38}$ R. Lipton, ${ }^{49}$ L. Lobo, ${ }^{42}$ A. Lobodenko, ${ }^{39}$ M. Lokajicek, ${ }^{11}$ A. Lounis, ${ }^{19}$ P. Love, ${ }^{41}$ H. J. Lubatti, ${ }^{78}$ L. Lueking, ${ }^{49}$ M. Lynker, ${ }^{54}$ A. L. Lyon, ${ }^{49}$ A. K. A. Maciel,${ }^{51}$ R. J. Madaras, ${ }^{45}$ P. Mättig, ${ }^{26}$ C. Magass ${ }^{21}$ A. Magerkurth, ${ }^{62}$ A.-M. Magnan, ${ }^{14}$ N. Makovec, ${ }^{16}$ P. K. Mal, ${ }^{29}$ H. B. Malbouisson, ${ }^{3}$ S. Malik,${ }^{58}$ V. L. Malyshev, ${ }^{35}$ H. S. Mao, ${ }^{6}$ Y. Maravin, ${ }^{49}$ M. Martens,${ }^{49}$ S. E. K. Mattingly, ${ }^{73}$ A. A. Mayorov, ${ }^{38}$ R. McCarthy, ${ }^{69}$ R. McCroskey, ${ }^{44}$ D. Meder ${ }^{24}$ A. Melnitchouk ${ }^{64}$ A. Mendes,${ }^{15}$ M. Merkin,${ }^{37}$ K. W. Merritt ${ }^{49}$ A. Meyer, ${ }^{21}$ J. Meyer ${ }^{22}$ M. Michaut, ${ }^{18}$ H. Miettinen, ${ }^{76}$ J. Mitrevski, ${ }^{67}$ J. Molina,${ }^{3}$ N. K. Mondal, ${ }^{29}$ R. W. Moore, ${ }^{5}$ G. S. Muanza, ${ }^{20}$ M. Mulders, ${ }^{49}$ Y. D. Mutaf, ${ }^{69}$ E. Nagy, ${ }^{15}$ M. Narain, ${ }^{60}$ N. A. Naumann, ${ }^{34}$ H. A. Neal, ${ }^{62}$ J. P. Negret, ${ }^{8}$ S. Nelson, ${ }^{48}$ P. Neustroev,${ }^{39}$ C. Noeding, ${ }^{23}$ A. Nomerotski, ${ }^{49}$ S. F. Novaes, ${ }^{4}$ T. Nunnemann, ${ }^{25}$ E. Nurse, ${ }^{43}$ V. O’Dell,${ }^{49}$ D. C. O’Neil,${ }^{5}$ V. Oguri, ${ }^{3}$ N. Oliveira, ${ }^{3}$ N. Oshima, ${ }^{49}$ G. J. Otero y Garzón, ${ }^{50}$ P. Padley ${ }^{76}$ N. Parashar, ${ }^{58}$ S. K. Park,${ }^{31}$ J. Parsons,${ }^{67}$ R. Partridge, ${ }^{73}$ N. Parua, ${ }^{69}$ A. Patwa,${ }^{70}$ G. Pawloski, ${ }^{76}$ P. M. Perea,${ }^{47}$ E. Perez,${ }^{18}$ P. Pétroff, ${ }^{16}$ M. Petteni, ${ }^{42}$ R. Piegaia, ${ }^{1}$ M.-A. Pleier,${ }^{68}$ 
P. L. M. Podesta-Lerma,${ }^{32}$ V. M. Podstavkov, ${ }^{49}$ Y. Pogorelov, ${ }^{54}$ A. Pompoš ${ }^{72}$ B. G. Pope,${ }^{63}$ W. L. Prado da Silva, ${ }^{3}$ H. B. Prosper, ${ }^{48}$ S. Protopopescu, ${ }^{70}$ J. Qian, ${ }^{62}$ A. Quadt, ${ }^{22}$ B. Quinn, ${ }^{64}$ K. J. Rani, ${ }^{29}$ K. Ranjan, ${ }^{28}$ P. A. Rapidis, ${ }^{49}$ P. N. Ratoff, ${ }^{41}$ S. Reucroft, ${ }^{61}$ M. Rijssenbeek, ${ }^{69}$ I. Ripp-Baudot,${ }^{19}$ F. Rizatdinova, ${ }^{57}$ S. Robinson, ${ }^{42}$ R. F. Rodrigues, ${ }^{3}$ C. Royon, ${ }^{18}$ P. Rubinov, ${ }^{49}$ R. Ruchti, ${ }^{54}$ V. I. Rud, ${ }^{37}$ G. Sajot, ${ }^{14}$ A. Sánchez-Hernández, ${ }^{32}$ M. P. Sanders, ${ }^{59}$ A. Santoro, ${ }^{3}$ G. Savage, ${ }^{49}$ L. Sawyer, ${ }^{58}$ T. Scanlon, ${ }^{42}$ D. Schaile, ${ }^{25}$ R. D. Schamberger ${ }^{69}$ H. Schellman, ${ }^{52}$ P. Schieferdecker, ${ }^{25}$ C. Schmitt, ${ }^{26}$ C. Schwanenberger, ${ }^{22}$ A. Schwartzman, ${ }^{66}$ R. Schwienhorst, ${ }^{63}$ S. Sengupta, ${ }^{48}$ H. Severini, ${ }^{72}$ E. Shabalina, ${ }^{50}$ M. Shamim, ${ }^{57}$ V. Shary, ${ }^{18}$ A. A. Shchukin, ${ }^{38}$ W. D. Shephard ${ }^{54}$ R. K. Shivpuri, ${ }^{28}$ D. Shpakov, ${ }^{61}$ R. A. Sidwell,${ }^{57}$ V. Simak, ${ }^{10}$ V. Sirotenko, ${ }^{49}$ P. Skubic,${ }^{72}$ P. Slattery, ${ }^{68}$ R. P. Smith, ${ }^{49}$ K. Smolek,,${ }^{10}$ G. R. Snow, ${ }^{65}$ J. Snow, ${ }^{71}$ S. Snyder, ${ }^{70}$ S. Söldner-Rembold, ${ }^{43}$ X. Song, ${ }^{51}$ L. Sonnenschein, ${ }^{17}$ A. Sopczak, ${ }^{41}$ M. Sosebee, ${ }^{74}$ K. Soustruznik,,${ }^{9}$ M. Souza, ${ }^{2}$ B. Spurlock, ${ }^{74}$ N. R. Stanton, ${ }^{57}$ J. Stark,${ }^{14}$ J. Steele, ${ }^{58}$ K. Stevenson, ${ }^{53}$ V. Stolin, ${ }^{36}$ A. Stone, ${ }^{50}$ D. A. Stoyanova, ${ }^{38}$ J. Strandberg, ${ }^{40}$ M. A. Strang, ${ }^{74}$ M. Strauss, ${ }^{72}$ R. Ströhmer, ${ }^{25}$ D. Strom, ${ }^{52}$ M. Strovink, ${ }^{45}$ L. Stutte, ${ }^{49}$ S. Sumowidagdo, ${ }^{48}$ A. Sznajder, ${ }^{3}$ M. Talby, ${ }^{15}$ P. Tamburello, ${ }^{44}$ W. Taylor, ${ }^{5}$ P. Telford, ${ }^{43}$ J. Temple,${ }^{44}$ M. Tomoto, ${ }^{49}$ T. Toole,${ }^{59}$ J. Torborg,${ }^{54}$ S. Towers, ${ }^{69}$ T. Trefzger, ${ }^{24}$ S. Trincaz-Duvoid, ${ }^{17}$ B. Tuchming, ${ }^{18}$ C. Tully, ${ }^{66}$ A. S. Turcot, ${ }^{43}$ P. M. Tuts,${ }^{67}$ L. Uvarov, ${ }^{39}$ S. Uvarov, ${ }^{39}$ S. Uzunyan, ${ }^{51}$ B. Vachon,${ }^{5}$ R. Van Kooten, ${ }^{53}$ W. M. van Leeuwen, ${ }^{33}$ N. Varelas, ${ }^{50}$ E. W. Varnes, ${ }^{44}$ A. Vartapetian, ${ }^{74}$ I. A. Vasilyev, ${ }^{38}$ M. Vaupel,${ }^{26}$ P. Verdier ${ }^{20}$ L. S. Vertogradov,${ }^{35}$ M. Verzocchi, ${ }^{59}$ F. Villeneuve-Seguier, ${ }^{42}$ J.-R. Vlimant, ${ }^{17}$ E. Von Toerne,${ }^{57}$ M. Vreeswijk, ${ }^{33}$ T. Vu Anh,${ }^{16}$ H. D. Wahl,${ }^{48}$ L. Wang, ${ }^{59}$ J. Warchol, ${ }^{54}$ G. Watts, ${ }^{78}$ M. Wayne, ${ }^{54} \mathrm{M}$. Weber, ${ }^{49} \mathrm{H}$. Weerts, ${ }^{63} \mathrm{M}$. Wegner, ${ }^{21} \mathrm{~N}$. Wermes, ${ }^{22}$ A. White,${ }^{74}$ V. White, ${ }^{49}$ D. Wicke, ${ }^{49}$ D. A. Wijngaarden, ${ }^{34}$ G. W. Wilson, ${ }^{56}$ S. J. Wimpenny, ${ }^{47} \mathrm{~J}$. Wittlin, ${ }^{60}$ M. Wobisch, ${ }^{49}$ J. Womersley, ${ }^{49}$ D. R. Wood, ${ }^{61}$ T. R. Wyatt, ${ }^{43}$ Q. Xu,${ }^{62}$ N. Xuan, ${ }^{54}$ S. Yacoob, ${ }^{52}$ R. Yamada, ${ }^{49}$ M. Yan, ${ }^{59}$ T. Yasuda, ${ }^{49}$ Y. A. Yatsunenko, ${ }^{35}$ Y. Yen, ${ }^{26}$ K. Yip, ${ }^{70}$ H. D. Yoo, ${ }^{73}$ S. W. Youn, ${ }^{52}$ J. Yu, ${ }^{74}$ A. Yurkewicz, ${ }^{69}$ A. Zabi, ${ }^{16}$ A. Zatserklyaniy, ${ }^{51}$ M. Zdrazil, ${ }^{69}$ C. Zeitnitz ${ }^{24}$ D. Zhang, ${ }^{49}$ X. Zhang, ${ }^{72}$ T. Zhao, ${ }^{78}$ Z. Zhao, ${ }^{62}$ B. Zhou, ${ }^{62}$ J. Zhu, ${ }^{69}$ M. Zielinski, ${ }^{68}$ D. Zieminska, ${ }^{53}$ A. Zieminski, ${ }^{53}$ R. Zitoun, ${ }^{69}$ V. Zutshi, ${ }^{51}$ and E. G. Zverev ${ }^{37}$

\title{
(D0 Collaboration)
}

\author{
${ }^{1}$ Universidad de Buenos Aires, Buenos Aires, Argentina \\ ${ }^{2}$ Centro Brasileiro de Pesquisas Físicas, LAFEX, Rio de Janeiro, Brazil \\ ${ }^{3}$ Universidade do Estado do Rio de Janeiro, Rio de Janeiro, Brazil \\ ${ }^{4}$ Instituto de Física Teórica, Universidade Estadual Paulista, São Paulo, Brazil \\ ${ }^{5}$ University of Alberta, Edmonton, Alberta, Canada, Simon Fraser University, Burnaby, British Columbia, Canada, \\ York University, Toronto, Ontario, Canada, \\ and McGill University, Montreal, Quebec, Canada \\ ${ }^{6}$ Institute of High Energy Physics, Beijing, People's Republic of China \\ ${ }^{7}$ University of Science and Technology of China, Hefei, People's Republic of China \\ ${ }^{8}$ Universidad de los Andes, Bogotá, Colombia \\ ${ }^{9}$ Center for Particle Physics, Charles University, Prague, Czech Republic \\ ${ }^{10}$ Czech Technical University, Prague, Czech Republic \\ ${ }^{11}$ Institute of Physics, Academy of Sciences, Center for Particle Physics, Prague, Czech Republic \\ ${ }^{12}$ Universidad San Francisco de Quito, Quito, Ecuador \\ ${ }^{13}$ Laboratoire de Physique Corpusculaire, IN2P3-CNRS, Université Blaise Pascal, Clermont-Ferrand, France \\ ${ }^{14}$ Laboratoire de Physique Subatomique et de Cosmologie, IN2P3-CNRS, Universite de Grenoble 1, Grenoble, France \\ ${ }^{15} C P P M$, IN2P3-CNRS, Université de la Méditerranée, Marseille, France \\ ${ }^{16}$ Laboratoire de l'Accélérateur Linéaire, IN2P3-CNRS, Orsay, France \\ ${ }^{17}$ LPNHE, IN2P3-CNRS, Universités Paris VI and VII, Paris, France \\ ${ }^{18}$ DAPNIA/Service de Physique des Particules, CEA, Saclay, France \\ ${ }^{19}$ IReS, IN2P3-CNRS, Université Louis Pasteur, Strasbourg, France, and Université de Haute Alsace, Mulhouse, France \\ ${ }^{20}$ Institut de Physique Nucléaire de Lyon, IN2P3-CNRS, Université Claude Bernard, Villeurbanne, France \\ ${ }^{21}$ III. Physikalisches Institut A, RWTH Aachen, Aachen, Germany \\ ${ }^{22}$ Physikalisches Institut, Universität Bonn, Bonn, Germany \\ ${ }^{23}$ Physikalisches Institut, Universität Freiburg, Freiburg, Germany \\ ${ }^{24}$ Institut für Physik, Universität Mainz, Mainz, Germany \\ ${ }^{25}$ Ludwig-Maximilians-Universität München, München, Germany \\ ${ }^{26}$ Fachbereich Physik, University of Wuppertal, Wuppertal, Germany \\ ${ }^{27}$ Panjab University, Chandigarh, India \\ ${ }^{28}$ Delhi University, Delhi, India \\ ${ }^{29}$ Tata Institute of Fundamental Research, Mumbai, India
}




\author{
${ }^{30}$ University College Dublin, Dublin, Ireland \\ ${ }^{31}$ Korea Detector Laboratory, Korea University, Seoul, Korea \\ ${ }^{32}$ CINVESTAV, Mexico City, Mexico \\ ${ }^{33}$ FOM-Institute NIKHEF and University of Amsterdam/NIKHEF, Amsterdam, The Netherlands \\ ${ }^{34}$ Radboud University Nijmegen/NIKHEF, Nijmegen, The Netherlands \\ ${ }^{35}$ Joint Institute for Nuclear Research, Dubna, Russia \\ ${ }^{36}$ Institute for Theoretical and Experimental Physics, Moscow, Russia \\ ${ }^{37}$ Moscow State University, Moscow, Russia \\ ${ }^{38}$ Institute for High Energy Physics, Protvino, Russia \\ ${ }^{39}$ Petersburg Nuclear Physics Institute, St. Petersburg, Russia \\ ${ }^{40}$ Lund University, Lund, Sweden, Royal Institute of Technology and Stockholm University, Stockholm, Sweden, \\ and Uppsala University, Uppsala, Sweden \\ ${ }^{41}$ Lancaster University, Lancaster, United Kingdom \\ ${ }^{42}$ Imperial College, London, United Kingdom \\ ${ }^{43}$ University of Manchester, Manchester, United Kingdom \\ ${ }^{44}$ University of Arizona, Tucson, Arizona 85721, USA \\ ${ }^{45}$ Lawrence Berkeley National Laboratory and University of California, Berkeley, California 94720, USA \\ ${ }^{46}$ California State University, Fresno, California 93740, USA \\ ${ }^{47}$ University of California, Riverside, California 92521, USA \\ ${ }^{48}$ Florida State University, Tallahassee, Florida 32306, USA \\ ${ }^{49}$ Fermi National Accelerator Laboratory, Batavia, Illinois 60510, USA \\ ${ }^{50}$ University of Illinois at Chicago, Chicago, Illinois 60607, USA \\ ${ }^{51}$ Northern Illinois University, DeKalb, Illinois 60115, USA \\ ${ }^{52}$ Northwestern University, Evanston, Illinois 60208, USA \\ ${ }^{53}$ Indiana University, Bloomington, Indiana 47405, USA \\ ${ }^{54}$ University of Notre Dame, Notre Dame, Indiana 46556, USA \\ ${ }^{55}$ Iowa State University, Ames, Iowa 50011, USA \\ ${ }^{56}$ University of Kansas, Lawrence, Kansas 66045, USA \\ ${ }^{57}$ Kansas State University, Manhattan, Kansas 66506, USA \\ ${ }^{58}$ Louisiana Tech University, Ruston, Louisiana 71272, USA \\ ${ }^{59}$ University of Maryland, College Park, Maryland 20742, USA \\ ${ }^{60}$ Boston University, Boston, Massachusetts 02215, USA \\ ${ }^{61}$ Northeastern University, Boston, Massachusetts 02115, USA \\ ${ }^{62}$ University of Michigan, Ann Arbor, Michigan 48109, USA \\ ${ }^{63}$ Michigan State University, East Lansing, Michigan 48824, USA \\ ${ }^{64}$ University of Mississippi, University, Mississippi 38677, USA \\ ${ }^{65}$ University of Nebraska, Lincoln, Nebraska 68588, USA \\ ${ }^{66}$ Princeton University, Princeton, New Jersey 08544, USA \\ ${ }^{67}$ Columbia University, New York, New York 10027, USA \\ ${ }^{68}$ University of Rochester, Rochester, New York 14627, USA \\ ${ }^{69}$ State University of New York, Stony Brook, New York 11794, USA \\ ${ }^{70}$ Brookhaven National Laboratory, Upton, New York 11973, USA \\ ${ }^{71}$ Langston University, Langston, Oklahoma 73050, USA \\ ${ }^{72}$ University of Oklahoma, Norman, Oklahoma 73019, USA \\ ${ }^{73}$ Brown University, Providence, Rhode Island 02912, USA \\ ${ }^{74}$ University of Texas, Arlington, Texas 76019, USA \\ ${ }^{75}$ Southern Methodist University, Dallas, Texas 75275, USA \\ ${ }^{76}$ Rice University, Houston, Texas 77005, USA \\ ${ }^{77}$ University of Virginia, Charlottesville, Virginia 22901, USA \\ ${ }^{78}$ University of Washington, Seattle, Washington 98195, USA \\ (Received 19 April 2005; published 5 October 2005)
}

A search for associated production of charginos and neutralinos is performed using data recorded with the D0 detector at a $p \bar{p}$ center-of-mass energy of $1.96 \mathrm{TeV}$ at the Fermilab Tevatron Collider. This analysis considers final states with missing transverse energy and three charged leptons, of which at least two are electrons or muons. No evidence for supersymmetry is found in a data set corresponding to an integrated luminosity of $320 \mathrm{pb}^{-1}$. Limits on the product of the production cross section and leptonic branching fraction are set. For the minimal supergravity model, a chargino lower mass limit of $117 \mathrm{GeV}$ at the $95 \%$ C.L. is derived in regions of parameter space with enhanced leptonic branching fractions. 
Supersymmetry (SUSY) predicts the existence of a new particle for each of the standard model particles, differing by half a unit in spin but otherwise sharing the same quantum numbers. No supersymmetric particles have been observed so far, and it is therefore generally assumed that they are heavier than their standard model partners. Experiments at the CERN LEP Collider have set lower limits on the masses of SUSY particles, excluding, in particular, charginos with masses below $103.5 \mathrm{GeV}$ as well as sleptons with masses below about $95 \mathrm{GeV}$ [1] in the framework of the minimal supersymmetric model (MSSM) [2]. Because of its high center-of-mass energy of $1.96 \mathrm{TeV}$, the Tevatron $p \bar{p}$ collider may produce SUSY particles with masses above these limits. A search for SUSY can be performed via the associated production of charginos and neutralinos. The lightest chargino $\tilde{\chi}_{1}^{ \pm}$and the second-lightest neutralino $\tilde{\chi}_{2}^{0}$ are assumed to decay via exchange of vector bosons or sleptons $\tilde{\ell}$ into the lightest neutralino $\tilde{\chi}_{1}^{0}$ and standard model fermions. Assuming conservation of $R$ parity, the lightest neutralino is stable and can only be detected indirectly.

This Letter reports on a search for $p \bar{p} \rightarrow \tilde{\chi}_{1}^{ \pm} \tilde{\chi}_{2}^{0}$ in final states with missing transverse energy and three charged leptons $(e, \mu$, or $\tau$ ), of which at least two are electrons or muons. The analysis is based on a data set recorded with the D0 detector between March 2002 and July 2004, corresponding to an integrated luminosity of $320 \mathrm{pb}^{-1}$. Previous searches in this channel have been performed by the CDF and D0 Collaborations with Tevatron Run I data [3].

The D0 detector consists of a central tracking system surrounded by a liquid-argon sampling calorimeter and a system of muon detectors [4]. Charged particles are reconstructed using multiple layers of silicon detectors as well as eight double layers of scintillating fibers in the $2 \mathrm{~T}$ magnetic field of a superconducting solenoid. The D0 calorimeter provides hermetic coverage up to pseudorapidities $|\eta| \approx 4$ in a semiprojective tower geometry with longitudinal segmentation. After passing through the calorimeter, muons are detected in three layers of tracking detectors and scintillation counters.

Events containing electrons or muons are selected for offline analysis by a real-time three-stage trigger system. A set of single and dilepton triggers has been used to tag the presence of electrons and muons based on their characteristic energy deposits in the calorimeter, the presence of high-momentum tracks in the tracking system, and hits in the muon detectors.

SUSY and standard model processes are modeled using the PYTHIA [5] Monte Carlo (MC) generator and a detailed simulation of the detector geometry and response based on GEANT [6]. Multiple interactions per crossing as well as pileup of signals in the calorimeter have been simulated. The MC events are then processed using the same reconstruction and analysis programs that are used for the data.
The background predictions are normalized using crosssection calculations at next-to-leading order (NLO) and next-to-NLO (for Drell-Yan production) with CTEQ6.1M [7] parton distribution functions (PDFs).

Background from multijet production is estimated from data. For this, samples dominated by multijet background have been defined that are identical to the search sample except for reversed lepton identification requirements. These samples are normalized at an early stage of the selection in a region of phase space dominated by multijet production.

Selection criteria are optimized to obtain the best average expected limit assuming that no signal will be observed. Limits are calculated at the $95 \%$ C.L. using the modified frequentist approach [8]. The optimization of selection cuts is based on signals inspired by minimal supergravity (mSUGRA) [2] with $\tilde{\chi}_{1}^{ \pm}, \tilde{\chi}_{2}^{0}$, and $\tilde{\ell}$ masses in the range 110-130 GeV. Because of the large production cross section and leptonic branching fraction via $\tilde{\ell}$ exchange, this mass range is of particular interest for a search in the trilepton channel. In the following discussion of the selection, as a representative example, a signal is used with common scalar mass $m_{0}=84 \mathrm{GeV}$, common fermion mass $m_{1 / 2}=176 \mathrm{GeV}$, ratio of Higgs vacuum expectation values $\tan \beta=3$, Higgs mass parameter $\mu>0$, and no slepton mixing, which corresponds to a $\tilde{\chi}_{1}^{ \pm}$mass of $110 \mathrm{GeV}$ and $\sigma \times \mathrm{BR}(3 \ell)=0.265 \mathrm{pb}$.

Four different selections are defined depending on the lepton content of the final state: two electrons plus lepton ( $e e \ell$ selection), two muons plus lepton $(\mu \mu \ell)$, two muons of the same charge $\left(\mu^{ \pm} \mu^{ \pm}\right)$, and one electron, one muon plus lepton $(e \mu \ell)$. The selection criteria are summarized in Table I and are discussed in more detail below.

Isolated electrons are identified based on their characteristic energy deposition in the calorimeter, including the transverse and longitudinal shower profile. In addition, it is required that a track point to this energy deposition and that its momentum and the calorimeter energy be consistent with the same electron energy. Remaining backgrounds from jets and photon conversions are suppressed based on the track activity around the track direction and by requiring the track associated with electron candidates to have associated hits in the innermost layers of the silicon detector.

Muons are reconstructed by finding tracks that point to patterns of hits in the muon system. Nonisolated muons from backgrounds with heavy-flavor jets are rejected by requiring the sum of track $p_{T}$ inside a cone with $\Delta \mathcal{R}=$ $\sqrt{(\Delta \phi)^{2}+(\Delta \eta)^{2}}=0.5$ around the muon direction to be less than $4 \mathrm{GeV}$ (loose muons) or less than $2.5 \mathrm{GeV}$ (tight muons). Tight muons are also required to have less than $2.5 \mathrm{GeV}$ deposited in the calorimeter in a hollow cone $0.1<\Delta \mathcal{R}<0.4$ around the muon direction.

Electron and muon reconstruction efficiencies have been measured using leptonic $Z$ boson decays collected by 
TABLE I. Selection criteria for the four analyses (all energies and momenta in $\mathrm{GeV}$, angles in radians); see text for further details.

\begin{tabular}{lccccc}
\hline \hline & Selection Cut & $e e \ell$ & $\mu \mu \ell$ & $\mu^{ \pm} \mu^{ \pm}$ & $e \mu \ell$ \\
\hline I & $p_{T}^{\ell 1}, p_{T}^{\ell 2}$ & $>12,>8$ & $>11,>5$ & $>11,>5$ & $>12,>8^{\mathrm{a}}$ \\
& $m_{\ell \ell}$ & $\in[18,60]$ & $\in[15,50]$ & $<80$ & $\ldots$ \\
II & $\Delta \phi_{\ell \ell}$ & $<2.9$ & $\ldots$ & $<2.7$ & $\ldots$ \\
& $\not_{T}$ & $>22$ & $>22$ & $>22$ & $\ldots$ \\
& $\operatorname{Sig}\left(\not \mathbb{E}_{T}\right)$ & $>8$ & $>8$ & $>8$ & $>15$ \\
& $m_{T}^{\min }$ & $>20$ & $>20$ & $\ldots$ & $\in[25,90]$ \\
III & jet-veto & $H_{T}<80$ & $\ldots$ & $\ldots$ & $H_{T}<40$ \\
IV & $p_{T}^{\ell 3}$ & $>4$ & $>3$ & $\ldots$ & $>7$ \\
V & $m_{\ell^{1,2} \ell^{3}}$ & $\ldots$ & $<70$ & $\notin[70110]^{\mathrm{b}}$ & $\notin[60120]$ \\
VI & $\left|\Sigma_{p_{T}}\right| / p_{T}^{\ell 3}$ & $\ldots$ & $\in[0.3,3.0]$ & $\ldots$ & $\ldots$ \\
VII & $\mathbb{E}_{T} \times p_{T}^{\ell 3}$ & $>220$ & $>150$ & $>300^{\mathrm{c}}$ & $\ldots$ \\
\hline \hline
\end{tabular}

${ }^{\mathrm{a}} p_{T}^{\ell 1}$ and $p_{T}^{\ell 2}$ are electron and muon $p_{T}$, respectively.

${ }^{\mathrm{b}}$ Opposite-sign muons only.

${ }^{\mathrm{c}}$ Using $p_{T}^{\ell 2}$ instead of $p_{T}^{\ell 3}$.

single-lepton triggers. The electron and muon trigger efficiencies have been measured in data and translate to an event trigger efficiency close to $100 \%$ (85\%) for signal events passing offline analysis requirements in the $e e \ell$, $e \mu \ell\left(\mu^{ \pm} \mu^{ \pm}, \mu \mu \ell\right)$ selections.

Each selection requires two identified leptons with minimum transverse momenta $p_{T}^{\ell 1}$ and $p_{T}^{\ell 2}$, using one loose muon for the $e \mu \ell$, one loose and one tight muon for the $\mu \mu \ell$, and two tight muons for the $\mu^{ \pm} \mu^{ \pm}$selection. Further selection cuts exploiting the difference in event kinematics and topology are applied as summarized in Table I. Di-electron and di-muon backgrounds from Drell-Yan and $Z$ boson production as well as multijet background are suppressed using cuts on the invariant dilepton mass $m_{\ell \ell}$ as well as the azimuthal opening angle $\Delta \phi_{\ell \ell}$, as illustrated in Fig. 1.

A further reduction in dilepton and multijet backgrounds can be achieved by requiring missing transverse energy $\boldsymbol{E}_{T}$ in an event. This is calculated as the vectorial sum of energy depositions in calorimeter cells and then adjusted using energy response corrections for reconstructed electrons, muons, and jets. Jets are defined using an iterative seed-based cone algorithm, clustering calorimeter energy within $\Delta \mathcal{R}=0.5$. For background events, an imbalance in transverse energy can be generated by mismeasurements of jet or lepton energies. Therefore, events in which the $\mathscr{E}_{T}$ direction is aligned with the lepton are removed using a cut on the minimum transverse mass $m_{T}^{\min }=\min \left(m_{T}^{\ell 1, \mathscr{L}_{T}}\right.$, $\left.m_{T}^{\ell 2, \not_{T}}\right)$ as shown in Fig. 1. In addition, events are rejected if they contain jets with transverse energies above $15 \mathrm{GeV}$ and have a small significance $\operatorname{Sig}\left(\mathscr{E}_{T}\right)$, which is defined by normalizing the $\not{E}_{T}$ to $\sigma\left(E_{T}^{j} \| \mathscr{E}_{T}\right)$, a measure of the jet energy resolution projected onto the $\mathbb{E}_{T}$ direction:

$$
\operatorname{Sig}\left(\mathscr{E}_{T}\right)=\frac{\mathscr{E}_{T}}{\sqrt{\sum_{\text {jets }} \sigma_{E_{T}^{j} \| \mathscr{E}_{T}}^{2}}} .
$$

Most of the remaining background from $t \bar{t}$ production can be rejected by removing events with large $H_{T}$, defined as
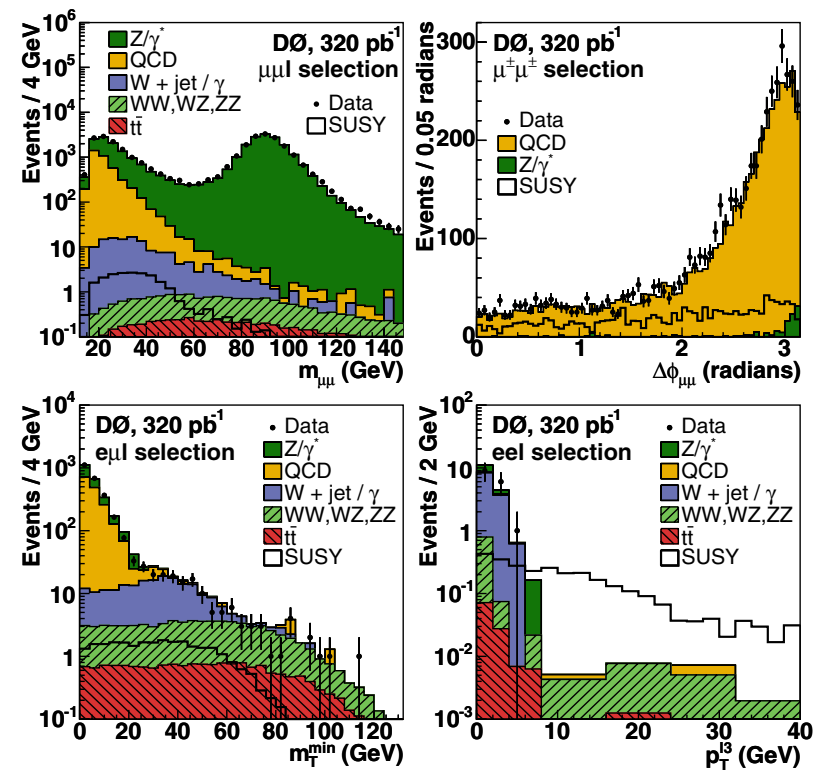

FIG. 1 (color online). Invariant di-muon mass $m_{\mu \mu}$ (upper left, $\mu \mu \ell$ selection), azimuthal di-muon opening angle $\Delta \phi_{\mu \mu}$ (upper right, $\mu^{ \pm} \mu^{ \pm}$selection), minimum transverse mass $m_{T}^{\min }$ (lower left, $e \mu \ell$ selection) and $p_{T}^{\ell 3}$, the transverse momentum of the third track (lower right, $e e \ell$ selection), for data (points), standard model backgrounds (shaded histograms), and SUSY signal (open histogram). 
TABLE II. Number of events observed in data and expected for background and reference signal (see text) at various stages of the selection, with statistical and systematic uncertainties added in quadrature. Each row corresponds to a group of cuts, as detailed in Table I.

\begin{tabular}{|c|c|c|c|c|c|c|c|c|c|c|c|c|}
\hline \multirow[t]{2}{*}{$\overline{\text { Cut }}$} & \multicolumn{3}{|c|}{ eee Selection } & \multicolumn{3}{|c|}{$\mu \mu \ell$ Selection } & \multicolumn{3}{|c|}{$\mu^{ \pm} \mu^{ \pm}$Selection } & \multicolumn{3}{|c|}{$e \mu \ell$ Selection } \\
\hline & Data & Backgrd. & Signal & Data & Backgrd. & Signal & Data & Backgrd. & Signal & Data & Backgrd. & Signal \\
\hline I & 33468 & $32000 \pm 3500$ & $8.8 \pm 0.8$ & 40489 & $40400 \pm 3100$ & $7.7 \pm 0.9$ & 201 & $235 \pm 22$ & $1.7 \pm 0.2$ & 2588 & $2600 \pm 290$ & $8.9 \pm 0.7$ \\
\hline II & 3921 & $3990 \pm 470$ & $6.2 \pm 0.6$ & 12520 & $11750 \pm 710$ & $6.1 \pm 0.7$ & 125 & $110 \pm 12$ & $1.3 \pm 0.1$ & $\cdots$ & $\cdots$ & $\cdots$ \\
\hline III & 46 & $45 \pm 12$ & $4.0 \pm 0.4$ & 135 & $182 \pm 38$ & $3.3 \pm 0.4$ & 7 & $5.7 \pm 1.6$ & $0.93 \pm 0.17$ & 95 & $95 \pm 11$ & $4.2 \pm 0.3$ \\
\hline IV & 1 & $0.47 \pm 0.28$ & $2.1 \pm 0.2$ & 16 & $24 \pm 7$ & $2.1 \pm 0.2$ & $\cdots$ & $\cdots$ & $\cdots$ & 5 & $4.1 \pm 0.6$ & $1.9 \pm 0.2$ \\
\hline $\mathrm{V}$ & $\cdots$ & $\cdots$ & $\cdots$ & 15 & $22.4 \pm 5.4$ & $1.8 \pm 0.2$ & 6 & $5.6 \pm 1.6$ & $0.90 \pm 0.17$ & 0 & $0.31 \pm 0.13$ & $1.6 \pm 0.1$ \\
\hline VI & $\cdots$ & $\cdots$ & $\cdots$ & 3 & $2.8 \pm 0.7$ & $1.4 \pm 0.2$ & $\cdots$ & $\cdots$ & $\cdots$ & $\cdots$ & $\cdots$ & $\cdots$ \\
\hline VII & 0 & $0.21 \pm 0.12$ & $1.9 \pm 0.2$ & 2 & $1.75 \pm 0.57$ & $1.3 \pm 0.2$ & 1 & $0.66 \pm 0.37$ & $0.70 \pm 0.14$ & $\cdots$ & $\cdots$ & $\cdots$ \\
\hline
\end{tabular}

the scalar sum of the transverse energies of all jets with $E_{T}>15 \mathrm{GeV}$.

The presence of the third lepton in signal events can be used for further separation from the background by requiring events to have a third, isolated, and well-measured track originating from the same vertex as the two identified leptons. To maximize signal yield, no additional lepton identification cuts are applied. The track (calorimeter) isolation conditions for this third track have been designed to be efficient for all lepton flavors, including hadronic decays of $\tau$ leptons, by allowing for tracks (energy deposits) inside an inner cone of $\Delta \mathcal{R}<0.1(\Delta \mathcal{R}<0.2)$. The distribution of the transverse momentum $p_{T}^{\ell 3}$ of the isolated track is shown in Fig. 1 for the eel selection. Except for $W Z$ events, a third track in background events generally originates from the underlying event or jets, and therefore tends to have very low transverse momentum. $W Z$ events are suppressed by removing events where the third track and one of the identified leptons have an invariant mass $m_{\ell^{1,2} \ell^{3}}$ consistent with the $Z$ boson mass $M_{Z}$. For the $\mu^{ \pm} \mu^{ \pm}$selection, backgrounds are low enough such that the requirement of a third track is not needed. Instead, background from $W Z \rightarrow \mu^{ \pm} \nu \mu^{ \pm} \mu^{\mp}$ is removed by vetoing events containing opposite-sign muons with an invariant mass close to $M_{Z}$. For the $\mu \mu \ell$ selection, on the other hand, a significant amount of multijet background remains; this is reduced by requiring that the vectorial sum $\left|\Sigma_{p_{T}}\right|$ of $\mathbb{E}_{T}$ and muon transverse momenta balances the transverse momentum of the third track.

Finally, a combined cut on the product of $\mathscr{E}_{T}$ and $p_{T}^{\ell 3}$ ( $p_{T}^{\ell 2}$ for $\mu^{ \pm} \mu^{ \pm}$) has been found to optimally reduce the remaining background, which tends to have both low $\mathbb{E}_{T}$ and low $p_{T}^{\ell 3}$. The expected numbers of events for background and the reference signal defined above are summarized in Table II at various stages of the selection. After all cuts, the expected background is dominated by multijet background (66\% and 53\% for the $\mu \mu \ell$ and $\mu^{ \pm} \mu^{ \pm}$selections, respectively) and di-boson backgrounds ( $80 \%$ and $88 \%$ for $e e \ell$ and $e \mu \ell$ ).
The estimates for expected numbers of background and signal events depend on numerous measurements that each introduce a systematic uncertainty: integrated luminosity $(6.5 \%)$, trigger efficiencies $(1 \%-2 \%)$, lepton identification and reconstruction efficiencies $(1 \%-2 \%)$, jet energy scale calibration in signal $(<4 \%)$ and background events $(7 \%-$ $20 \%$ ), lepton and track momentum calibration (1\%), detector modeling ( $2 \%)$, PDF uncertainties $(<4 \%)$, and modeling of multijet background $(4 \%-40 \%)$. The uncertainties quoted in Table II in addition contain the statistical uncertainty due to limited MC statistics, which is the dominant uncertainty for backgrounds from $W$ and $Z$ boson production.

As can be seen in Table II, the numbers of events observed in the data are in good agreement with the expectation from standard model processes at all stages of the selection. Combining all four selections, a total background of $2.93 \pm 0.54$ (stat) \pm 0.57 (syst) events is expected after all cuts, while 3 events are observed in the data.

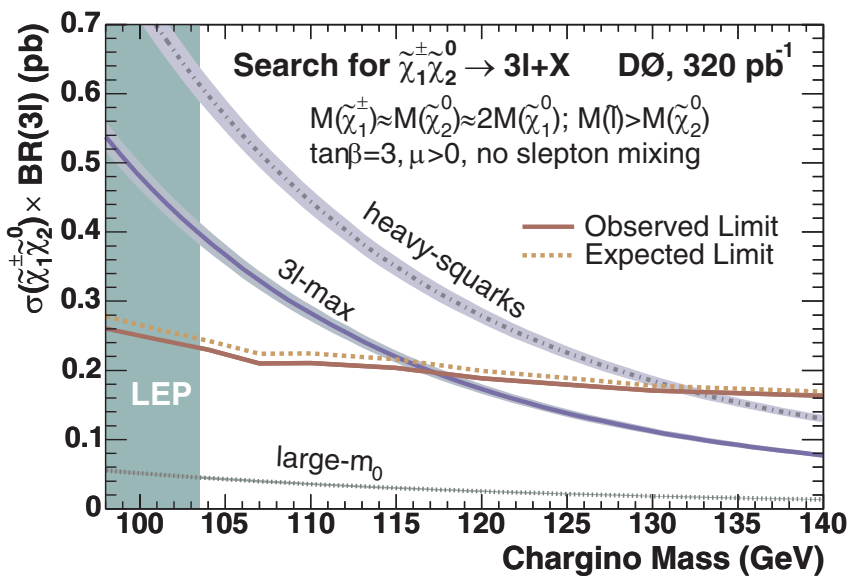

FIG. 2 (color online). Limit on $\sigma \times \mathrm{BR}(3 \ell)$ as a function of $\tilde{\chi}_{1}^{ \pm}$mass, in comparison with the expectation for several SUSY scenarios (see text). PDF and renormalization/factorization scale uncertainties are shown as shaded bands. 


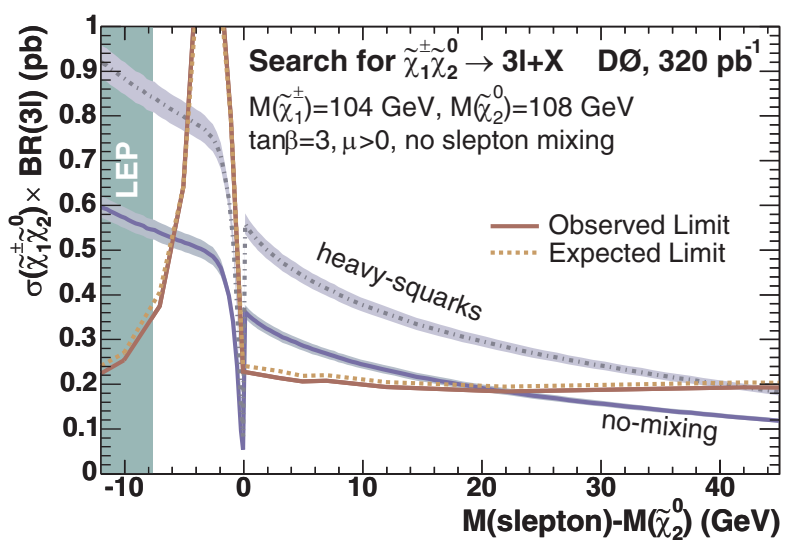

FIG. 3 (color online). Limit on $\sigma \times \mathrm{BR}(3 \ell)$ as a function of the mass difference between $\tilde{\ell}$ and $\tilde{\chi}_{2}^{0}$, in comparison with the expectation for the MSSM (no mixing) and the heavy-squarks scenario (see text). PDF and renormalization/factorization scale uncertainties are shown as shaded bands. BR $(3 \ell)$ drops sharply at $m_{\tilde{\ell}} \lesssim m_{\tilde{\chi}_{2}^{0}}$ as the phase space for two-body decays into real sleptons is minimal.

Since no evidence for associated production of charginos and neutralinos is observed, an upper limit on the product of production cross section and leptonic branching fraction $\sigma \times \mathrm{BR}(3 \ell)$ is extracted from this result. As mentioned above, information from the four selections is combined using the modified frequentist approach, taking into account correlated errors. The small fraction of signal events that is selected by more than one selection is assigned to the selection with the largest signal-tobackground ratio and removed from all others.

The expected and observed limits are shown in Figs. 2 and 3 as a function of $\tilde{\chi}_{1}^{ \pm}$mass and of the difference between $\tilde{\chi}_{2}^{0}$ and $\tilde{\ell}$ masses, respectively. This result improves significantly the upper limit of about $1.5 \mathrm{pb}$ set by the D0 Run I analysis [3]. Assuming the mSUGRAinspired mass relation $m_{\tilde{\chi}_{1}^{ \pm}} \approx m_{\tilde{\chi}_{2}^{0}} \approx 2 m_{\tilde{\chi}_{1}^{0}}$ as well as degenerate slepton masses $m_{\tilde{\ell}}$ (no slepton mixing), the limit on $\sigma \times \mathrm{BR}(3 \ell)$ is a function of $m_{\tilde{\chi}_{1}^{ \pm}}$and $m_{\tilde{\ell}}$, with a relatively small dependence on the other SUSY parameters. This result can therefore be interpreted in more general SUSY scenarios, as long as the above mass relations are satisfied and $R$ parity is conserved. The leptonic branching fractions of $\tilde{\chi}_{1}^{ \pm}$and $\tilde{\chi}_{2}^{0}$ depend on the relative contribution from the $\tilde{\ell}$ - and $W / Z$-exchange graphs, which varies as a function of $m_{\tilde{\ell}} . W / Z$ exchange is dominant at large $m_{\tilde{\ell}}$, resulting in relatively small leptonic branching fractions (large- $m_{0}$ scenario). The leptonic branching fraction for three-body decays is maximally enhanced for $m_{\tilde{\ell}} \geq m_{\tilde{\chi}_{2}^{0}}$ ( $3 \ell$-max scenario). Decays into leptons can even be dominant if sleptons are light enough that two-body decays are possible. In the latter case, one of the leptons from the $\tilde{\chi}_{2}^{0}$ decay can have a very low transverse momentum if $\left|m_{\tilde{\ell}}-m_{\tilde{\chi}_{2}^{0}}\right|$ is small. In this region, only the $\mu^{ \pm} \mu^{ \pm}$selec- tion remains efficient, leading to a higher limit for $-6 \lesssim$ $m_{\tilde{\ell}}-m_{\tilde{\chi}_{2}^{0}}<0 \mathrm{GeV}$ (see Fig. 3). In addition, the $\tilde{\chi}_{1}^{ \pm} \tilde{\chi}_{2}^{0}$ production cross section depends on the squark masses due to the negative interference with the $t$-channel squark exchange. Relaxing scalar mass unification, the cross section is maximal in the limit of large squark masses (heavysquarks scenario). The NLO prediction [9] for $\sigma \times$ BR $(3 \ell)$ for these reference scenarios is shown in Figs. 2 and 3. The cross-section limit set in this analysis corresponds to a chargino mass limit of $117 \mathrm{GeV}(132 \mathrm{GeV})$ in the $3 \ell$-max (heavy-squarks) scenario, which improves on the mass limit set by chargino searches at LEP.

In summary, no evidence for supersymmetry is observed in a search for associated chargino and neutralino production in trilepton events. Upper limits on the product of cross section and leptonic branching fraction are set, which improve previous limits set with the Run I data set. Chargino mass limits beyond the reach of LEP chargino searches are derived for several SUSY reference scenarios with enhanced leptonic branching fractions.

We thank the staffs at Fermilab and collaborating institutions, and acknowledge support from the DOE and NSF (USA), CEA and CNRS/IN2P3 (France), FASI, Rosatom and RFBR (Russia), CAPES, CNPq, FAPERJ, FAPESP and FUNDUNESP (Brazil), DAE and DST (India), Colciencias (Colombia), CONACyT (Mexico), KRF (Korea), CONICET and UBACyT (Argentina), FOM (The Netherlands), PPARC (United Kingdom), MSMT (Czech Republic), CRC Program, CFI, NSERC and WestGrid Project (Canada), BMBF and DFG (Germany), SFI (Ireland), A. P. Sloan Foundation, Research Corporation, Texas Advanced Research Program, Alexander von Humboldt Foundation, and the Marie Curie Program.

*Visitor from University of Zurich, Zurich, Switzerland.

[1] LEPSUSYWG, ALEPH, DELPHI, L3, and OPAL experiments, notes LEPSUSYWG/01-03.1 and 04-01.1, http:// lepsusy.web.cern.ch/lepsusy/Welcome.html.

[2] H. P. Nilles, Phys. Rep. 110, 1 (1984).

[3] B. Abbott et al. (D0 Collaboration), Phys. Rev. Lett. 80, 1591 (1998); F. Abe et al. (CDF Collaboration), Phys. Rev. Lett. 80, 5275 (1998).

[4] V. Abazov et al. (D0 Collaboration), Nucl. Instrum. Methods (to be published); T. LeCompte and H. T. Diehl, Annu. Rev. Nucl. Part. Sci. 50, 71 (2000).

[5] T. Sjöstrand et al., Comput. Phys. Commun. 135, 238 (2001).

[6] R. Brun and F. Carminati, CERN Program Library Long Writeup W5013, 1993 (unpublished).

[7] J. Pumplin et al., J. High Energy Phys. 07 (2002) 12; D. Stump et al., J. High Energy Phys. 10 (2003) 046.

[8] T. Junk, Nucl. Instrum. Methods Phys. Res., Sect. A 434, 435 (1999).

[9] W. Beenakker et al., Phys. Rev. Lett. 83, 3780 (1999). 